\title{
Touch to Play - Exploring Touch-Based Mobile Interaction with Public Displays
}

\author{
Gregor Broll ${ }^{1}$, Roman Graebsch ${ }^{2}$, Maximilian Scherr ${ }^{3}$, Sebastian Boring ${ }^{3,4}$, Paul Holleis ${ }^{1}$, Matthias Wagner ${ }^{1}$ \\ ${ }^{1}$ DOCOMO Euro-Labs, Munich, Germany \\ \{broll, holleis, wagner\}@docomolab-euro.com \\ 2 IT University of Copenhagen, Copenhagen, Denmark \\ r.graebsch@googlemail.com \\ ${ }^{3}$ Ludwig-Maximilians Universität München, Munich, Germany \\ scherr@cip.ifi.lmu.de, sebastian.boring@ifi.lmu.de \\ ${ }^{4}$ Department of Computer Science, University of Calgary, Calgary, Canada \\ sebastian.boring@ucalgary.ca
}

\begin{abstract}
Mobile interactions with public displays are often indirect and not very convenient for multiple users at the same time. In this paper we use the physical, touch-based interaction with Near Field Communication (NFC) to investigate direct mobile interactions with public displays for multiple users. For that purpose, we adopt the Whack-a-Mole game for dynamic NFC-displays, which combine the physical interaction with NFCtagged objects and the visual output capabilities of public displays. We show that a grid of NFC-tags can be used to implement direct mobile interaction with public displays in general and a highly interactive multiplayer game in particular. Our study shows that users appreciate this physical, NFC-based mobile interaction, although technical advances are still necessary in order to improve its recognition rate of about $70 \%$. The study also indicates that users are willing to interact with large displays in public, but prefer private or semi-public places, where their interactions attract less attention.
\end{abstract}

\section{INTRODUCTION}

Large public displays have literally become one of the most visible manifestations of Ubiquitous Computing in everyday environments, including pedestrian areas, shopping malls, train stations or airports. In order to enable the interaction with public displays and their contents, many approaches take advantage of mobile devices and their input features, e.g. keys, joysticks, cameras, touch-screens, accelerometers or other sensors [1], [2]. Still, these interactions are mostly indirect and can even be constrained by narrow keyboards, fiddly controllers or imprecise sensors. This indirectness of mobile interaction with public displays is further increased by the distance between mobile devices and screens as well as the size of the latter. Examples range from large screens in stadiums or for public viewing to TV-size screens in bars or shops. Because of this indirectness, mobile interaction with public displays is often hard to individualize for different users and is not very convenient for multiple users.

In this paper we use the physical, touch-based interaction with Near Field Communication (NFC) [20] to investigate direct interactions between mobile devices and public displays. In order to implement and evaluate this interaction, we adopt the Whack-a-Mole game for dynamic NFC-displays. This technology uses a grid of NFC-tags as a physical user interface (UI) that users can touch with their mobile devices to manipulate an application UI that is projected onto the physical UI (Fig. 1). The physical interaction between individual mobile devices and NFC-tags enables a more direct interaction between mobile devices, large screens and their contents than public displays or touch screens.

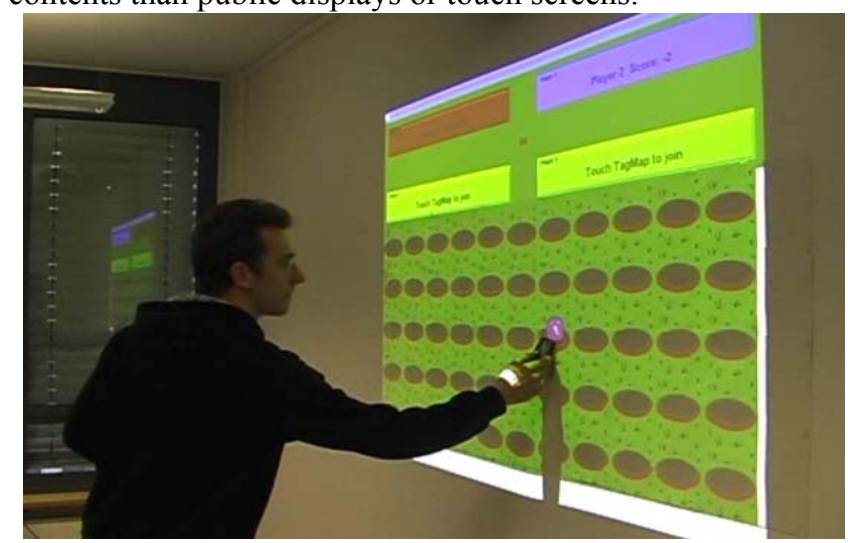

Fig. 1. Direct, touch-based interaction with a dynamic NFC-display

We use a gaming scenario to push the technical boundaries of mobile interaction with dynamic NFC-displays and to assess its accuracy, reliability and speed in a playful way. That way, we can also evaluate how the physical interaction with NFC-based UIs can improve the directness, accessibility and usability of mobile interactions with public displays. Finally, this approach allows us to assess the appropriateness of dynamic NFC-displays as a technology for direct mobile interaction with public displays in general and mobile gaming in particular. Our study shows that users appreciate the playfulness and ease of use of NFC-based mobile interaction although the tag recognition rate of about $70 \%$ leaves room for technical improvements. The study also indicates that users are willing to interact with large displays in public, but prefer private or semi-public places, where their interactions attract less attention. 
The next section gives an overview of related work about mobile interaction with NFC, physical UIs and public displays. Section 3 shows how dynamic NFC-displays can be used to implement direct, physical interactions between mobile devices and public displays for multiple users, which will be evaluated in section 4 . Section 5 discusses the results and concludes this paper.

\section{RELATED WORK}

Near Field Communication (NFC) is a radio-based technology for short-range data exchange between reading devices, e.g. mobile phones, and passive wireless tags. It is closely related to Radio Frequency Identification (RFID), but better tailored to support different interaction modalities (read/write, tag emulation, P2P) in mobile devices [20]. Mobile interaction with NFC and RFID has considerably evolved since 1999, when Want et al. [19] tagged everyday objects such as books or business cards with RFID-tags to link them with electronic documents or email-addresses. Since then, an increasing number of (commercial) mobile applications has taken advantage of the simple, touch-like interaction between NFC-enabled mobile devices, readers, tags or smartcards to facilitate payment (e.g. i-mode FeliCa [9]), ticketing (e.g. Oyster Card [10]), access control, home care or entertainment [17]. In Ubicomp research, NFC and RFID are used to tag physical objects in order to identify them, to link them with digital resources like information or services and to facilitate the interaction with them [4], [14].

While most applications use simple interactions with single NFC-tags, some applications map features and options to multiple tags on physical objects, turning them into physical UIs that complement mobile UIs and adopt some of their features. Examples are posters that comprise multiple tags to let users invoke Web Services for mobile ticketing [4]. Sanchez et al. [15] use a tagged panel to operate a multimedia player whose controls are mapped to RFID-tags that users can touch with their mobile devices. Reilly et al. [13] use RFIDtags on the back of maps to interact with points-of-interests.

In the next step of their evolution, physical UIs are covered with a grid of NFC-tags to provide a completely interactive surface for the manipulation of dynamic application UIs. Opposite to static posters, these tags do not refer to specific items of information, e.g. POIs on a map, but merely indicate their position in the grid. That way, tags can be dynamically mapped to the UI elements of different applications. Vetter et al. [18] and Hardy et al. [8] have created and refined the first dynamic NFC-displays that use a grid of NFC-tags for the interaction with an application UI that is projected onto this interactive surface. Similarly, Ramírez-González et al. [11] have combined a grid of NFC-tags and a projected application UI to build an interactive NFC-panel. Seewoonauth et al. [16] use a grid of tags on the back of a laptop display to enable direct, touch-based interactions.

RFID and NFC can be used to bring features of videogames to the real world, respectively to tag physical objects in order to include them in digital games, creating a mixed reality experience [6]. PAC-LAN [12] for example brings the classic Pacman videogame to the real world setting of a student apartment complex. The pills of the original game have been replaced by plastic discs with RFID-tags that players have to touch in order to collect points and to see the position of other players on their mobile devices. In addition, all players (PACLAN and ghosts) wear tags that have to be touched to "kill" a player. Mobspray [7] uses postcards with RFID-tags to emulate the tagging of physical locations with graffiti. Users can leave their graphical marks on these locations by writing onto the RFID-tags with their mobile phones instead of using a spray can. A tag stores the names of the last five visitors. Users can add their own names and graphical tags to this list or view the graphical tags of previous visitors by connecting to an online database. Finally, Mobhunt [6] emulates classic treasure hunt games, but uses RFID-tags to store clues about the hidden "treasure" of the game.

Mobile interaction with public displays often takes advantage of the various input features of mobile devices: Sweep [1] uses optical-flow recognition of phonecam images to recognize the movement of a mobile phone and to control a cursor on a display. With Point \& Shoot [1], users take pictures of a grid of visual markers to identify and select objects on a display. Boring et al. [2] compare the techniques Scroll (using input from the keys or joysticks of mobile phones), Tilt (sensed by an accelerometer) and Move (using optical-flow image recognition) to control a cursor on a display. A common denominator of all these input features is their indirect interaction with public displays resulting in virtual pointers shown for each user.

\section{The Whack-A-Mole Prototype}

The Whack-a-Mole game is well suited to explore direct, NFC-based mobile interaction with public displays [5]. The simple gameplay - hitting moles with a mallet - is driven by physical interaction and can take advantage of the touch-based interaction with dynamic NFC-displays and its support for individual and multi-user interactions. This and other games on dynamic NFC-displays can be set up at public locations like underground stations, arcades, shopping malls or pubs, where they encourage spontaneous interactions between people, e.g. playing a game while waiting for the train.

\section{A. Dynamic NFC-Displays}

Dynamic NFC-displays combine the simplicity and directness of physical interaction with NFC-tagged objects and the visual output capabilities of large screens (Fig. 2). They comprise a server that manages the logic of an application and projects its graphical UI onto a grid of NFCtags that serves as a physical UI. Users can manipulate the contents of the projected application UI by touching the tags of the physical UI with an NFC-enabled mobile device. A mobile client returns the position of the tags in the grid to the server which updates the application UI according to the interaction. 


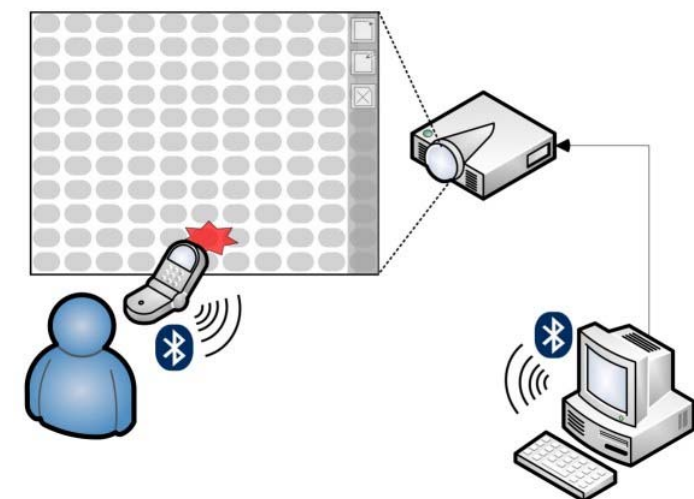

Fig. 2. Basic setup of a dynamic NFC-display including the server, the projector, the physical UI with a grid of NFC-tags and the mobile device

Dynamic NFC-displays are a prototyping technology that can be used to implement direct, touch-based interactions between mobile devices and large screens. Touching the tags of the NFC-grid with a mobile device allows a more physical and direct interaction between mobile devices and items on a screen than public displays or touch screens. Examples for such interactions are the selection of specific items, their direct manipulation (e.g. drag\&drop) or their transfer between mobile devices and displays. While some approaches implement direct interactions with the content of large screens, e.g. [3], public displays usually do not support physically close, e.g. touch-based, interactions with their content. Touch screens provide direct interactions with screen items, but not between mobile devices and items.

The direct interaction between individual mobile devices and NFC-tags also facilitates the identification of different users. That way, interactions can be better adapted to different users and can also support multiple users at the same time. Although mobile interactions with public display and touch screens can also identify different users, many of these approaches are not convenient for multi-user interaction.

\section{B. Technical Setup}

The Whack-a-Mole prototype comprises a physical UI that is big enough to let several users interact with it at the same time. It is composed of reconfigurable tiles that have the size of a DIN A4 sheet and contain 5 by 8 Mifare NFC-tags each (Fig. 3). The complete physical UI of the prototype consists of 4 by 6 tiles, resulting in a total size of $164 \mathrm{~cm}$ by $69.5 \mathrm{~cm}$ and a total of 960 NFC-tags (Fig. 4).

We decided to use a special brand of NFC-tags with a round shape, which is not perfect for building a completely interactive surface without gaps between adjacent tags. However, the comparison with other brands of (rectangular) tags showed that tags of this brand were technically more reliable. Therefore, the tags on the tiles and the assembled tiles overlap to form a completely interactive surface without significant gaps between tiles or tags. We tested different gridsizes to balance the arrangement of NFC-tags and the degree to which they can overlap. The diameter of the NFC-tags we used is $42 \mathrm{~mm}$ and we ended up with a grid-size of $35 \mathrm{~mm}$, resulting in an interactive surface that is almost completely covered with NFC-tags. That way, users can touch the physical UI in any place and usually hit an NFC-tag. The assembled physical UI was covered with a blank sheet of paper to provide a smooth surface that does not indicate the position of the single tags in the grid.

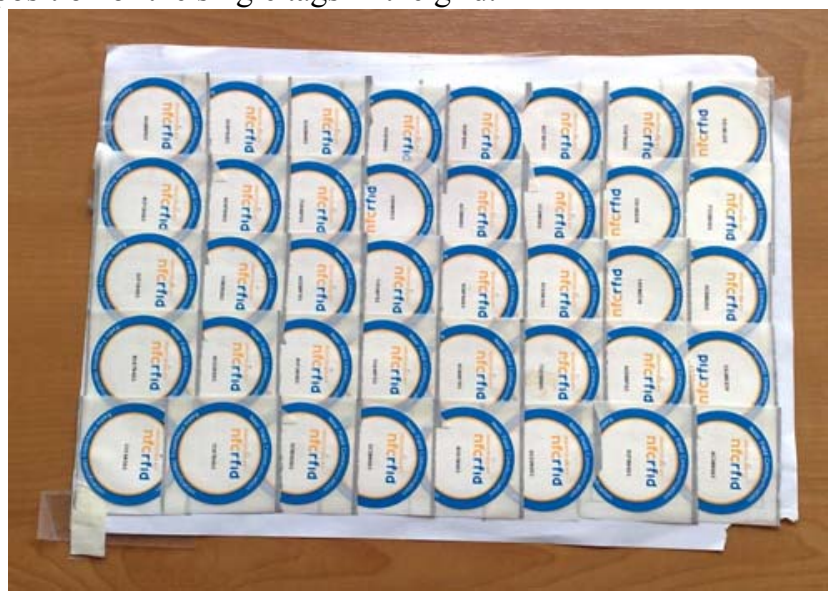

Fig. 3. Backside of a tile of the NFC-grid with 8 by 5 overlapping tags

A ceiling-mounted short throw projector projects the application UIs onto the physical UI from above users, producing less shadow than regular projectors that may handicap the interaction (Fig. 4). The hardware setup is completed by a laptop that runs the Java SE game server and several NFC-enabled Nokia 6212 phones that run a mobile Java ME client for the interaction with the physical UI and the manipulation of the application UI. The phones serve as smart pointing devices that read the tags of the physical UI, communicate with the application server via Bluetooth, provide feedback and identify users, especially during multiuser interactions.

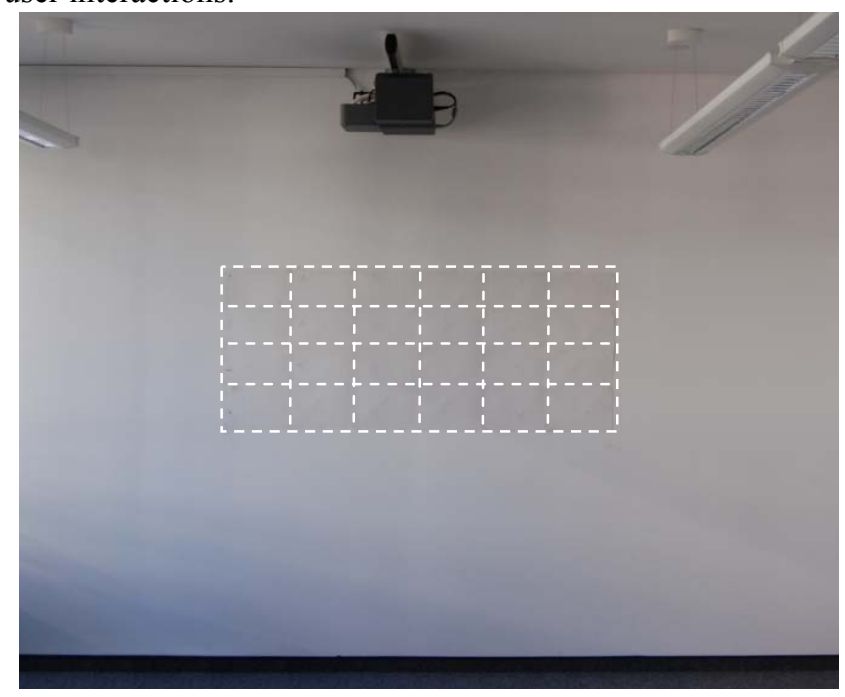

Fig. 4. Complete setup of the NFC-grid and the short throw projector for the Whack-a-Mole prototype (white lines mark the position of tiles in the grid)

Reading devices, NFC-tags and their assembly in physical UIs affect the accuracy, speed and smoothness of NFC-based mobile interaction. The Nokia 6212 that we used for the 
interaction with the NFC-tags can only read one tag at a time. Reading a tag causes a short, but considerable delay of about 0.5 seconds. These aspects implicate a discrete interaction with the NFC-tags of the physical UI and prohibit continuous readings of tags, e.g. for gestures, or reading multiple tags at the same time. Using smaller tags to increase the resolution of the physical UI might support more precise interactions. However, the effectiveness of a higher resolution for NFCbased mobile interaction also depends on the size of the reading unit, respectively the width of the mobile phone that may occlude tags during the interaction with them. Therefore, we stuck with tags whose diameter is about the width of the Nokia 6212.

\section{Game Design and Gameplay}

The objective of the original Whack-a-Mole game is to hit moles with a toy mallet while they pop up from holes in a wooden cabinet. The Whack-a-mole prototype for dynamic NFC-displays follows the design of the original game. Its game UI (Fig. 1) contains four status bars, a countdown for the playing time and the gaming area with holes from where the moles pop up during the game. While this area is projected onto the grid of NFC-tags, the status bars are projected above it and are not interactive. After a player has started a new game from his mobile device, up to three other players can join it by touching the dynamic NFC-display. Each player is assigned a color which is indicated in his status bar.

During the game, moles randomly rise and recede from their holes. Players can hit them by touching the NFC-tags beneath them with their NFC-enabled mobile phones (Fig. 5). A successful hit is indicated by visual feedback on the game UI and by vibration on the mobile device. In order to win the game, the players have to hit as many moles as possible until the countdown runs out after 100 seconds. The moles are colored and players earn one credit for hitting one of them. To make the game more challenging, a player loses a credit if somebody hits a mole of his color. That way, players not only have to hit moles of other players, but also have to guard their own moles against them (Fig. 6).

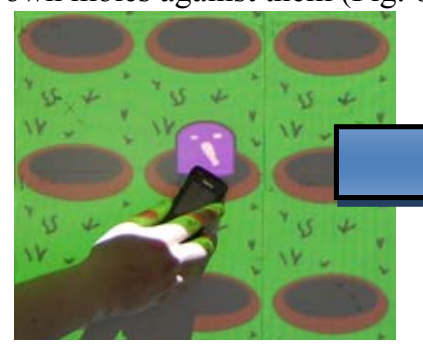

a)

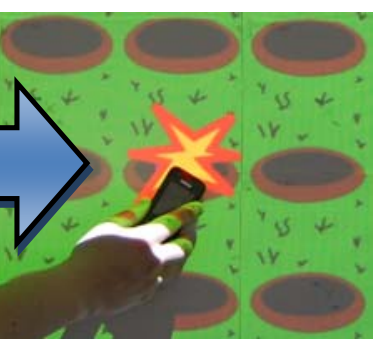

b)
Fig. 5. Moles pop up from their virtual holes (a) and can be hit with NFCenabled phones (b) to earn credits

The Whack-a-Mole prototype shows how mobile interaction with public displays can benefit from the physical, touch-based interaction with NFC to improve its accessibility and usability. The interaction between mobile devices and the dynamic NFC-display enables direct manipulations of display content and application features. Users do not have to struggle with Bluetooth to pair mobile devices and displays manually or master the input features of the former. Instead, users can simply touch the screen with their mobile devices to initiate the Bluetooth connection with it automatically, to manipulate its content and to personalize their actions, especially during interactions with other players (Fig. 6).

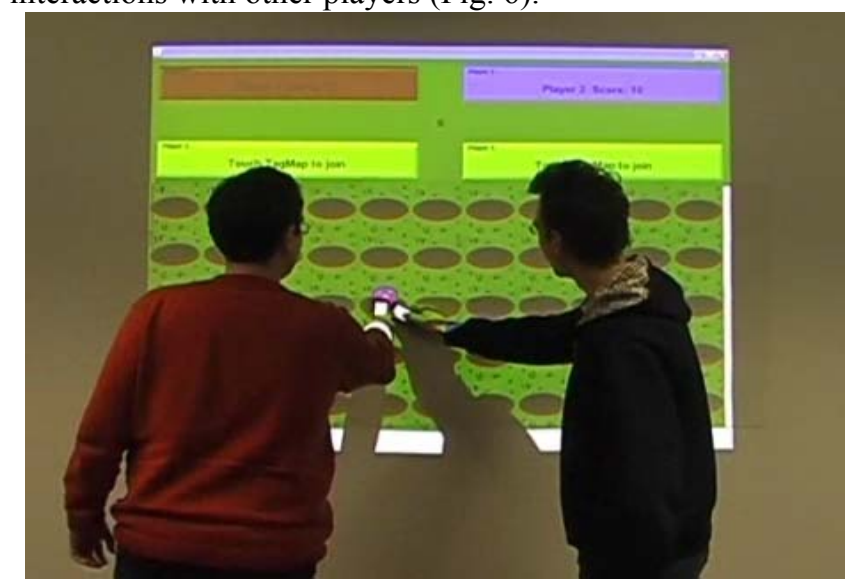

Fig. 6. Increased competition between multiple players

\section{USER STUDY AND EVALUATION}

Using the Whack-a-Mole prototype, we conducted a user study to evaluate direct, touch-based interaction with public displays as well as the accuracy and usability of mobile interaction with NFC-based physical UIs.

\section{A. Experimental Design, Participants and Procedure}

The study was conducted in our lab with 18 subjects (15 male, 3 female) with an average age of 30 years (from 24 to 52 ). The subjects were recruited from our lab and a university where most of them work as researchers or students. Consequently, most subjects had a background in computer sciences and rated their general technical expertise and their expertise with mobile devices with high average values - both 3.9 on a Likert-scale from 1 ("little experience") to 5 ("great experience"). However, most subjects were not familiar with NFC - 10 of them have heard about it before the study but only 3 have actually used it.

During the study, the subjects played three rounds with the Whack-a-Mole prototype. The first round introduced the novel interaction with NFC and familiarized the subjects with it. The subjects played the second round alone and competed with the investigator in the third round to test multi-user interaction. The subjects were recorded on video to assess their interactions with the dynamic NFC-display in a poststudy analysis. In the end, the subjects filled out a questionnaire to assess the prototype, its technology and the interaction with it.

\section{B. Results}

One goal of the study was to assess the accuracy of the tag recognition during the interaction with the grid of overlapping NFC-tags. During the video analysis, we counted the number of total and successful attempts to hit moles by touching the 
NFC-tags beneath them. Out of the total of 641 hits, 440 hits were successful while 201 hits failed due to technical issues while trying to read a tag, resulting in a mean failure rate of $31.4 \%$. Reasons for failed hits were hitting the small gaps or the overlapping sections between adjacent tags. In most cases, the phone should be able to recognize at least one tag, but the analysis showed that in 3 out of 10 tries, the phone had problems to recognize overlapping tags.

The questionnaire also evaluated the perceived performance of the tag recognition and its impact on the game, asking the subjects to rate different statements on a Likert-scale from 1 ("disagree") to 5 ("agree"). The subjects were undecided whether the recognition of the NFC-tags was accurate (mean $=3.1$; standard deviation $=0.7)$ or fast enough for playing the game $(\mathrm{m}=3.2 ; \mathrm{sd}=1.1)$ or whether the interaction with the tags produced many errors $(\mathrm{m}=3.0 ; \mathrm{sd}=0.8)$. When asked about problems during the game, half of the subjects mentioned that the recognition of the tags was sometimes inaccurate or that they missed tags. One way to improve the accuracy of the tag recognition and to decrease the number of failed hits would be to mark the position of the tags on the physical UI, e.g. by drawing the grid on its front. The subjects slightly agreed $(\mathrm{m}=3.6, \mathrm{sd}=1.2)$ that this design could indeed facilitate the interaction with the NFC-tags.

Despite the inaccuracy of the tag recognition, the subjects considered the touch-based interaction to be fun $(\mathrm{m}=4.2 ; \mathrm{sd}$ $=0.8)$ and easy to learn $(\mathrm{m}=4.6 ; \mathrm{sd}=0.7)$. They thought that it made the game more immersive $(\mathrm{m}=3.8$; $\mathrm{sd}=0.6)$ and easier to use $(\mathrm{m}=3.9 ; \mathrm{sd}=0.8)$, but not physically exhaustive $(\mathrm{m}=2.6 ; \mathrm{sd}=1.1)$, maybe because of the short time they played the game. They also slightly agreed that the competitive interaction with other players improved the gaming experience $(\mathrm{m}=3.5 ; \mathrm{sd}=1.2)$. The subjects clearly disagreed that the projection of the game UI affected the interaction in a negative way $(\mathrm{m}=1.8 ; \mathrm{sd}=1.0)$ which advocates the use of a ceiling-mounted short focus projector instead of a regular projector. The subjects were undecided whether the interaction with the physical UI was easier than the interaction on a mobile device $(\mathrm{m}=3.1$; $\mathrm{sd}=0.7)$. The size of the display, the easy and intuitive physical interaction and the multi-player support were seen as advantages of interacting with the NFC-display. The lack of accuracy was confirmed as the greatest disadvantage.

Physical interactions with large displays are clearly visible and attract the attention of other people. On the one hand, this visibility may increase the interest of potential users and encourage spontaneous interactions with public displays. On the other hand, it may repel potential users who prefer less attention and more privacy. The last part of the questionnaire asked the subjects where and with whom they would play the Whack-a-Mole game: In private places (e.g. at home), most of the subjects would play the game with friends (14 votes), but understandably not with strangers (0). 4 subjects would not play the game at all. In semi-public places that are open to the public but usually visited by specific groups of people (e.g. pubs, arcades), 13 subjects would again play the game with friends. 2 subjects would not play the game at all, but 8 subjects were also open to play with strangers. The results for playing the game in public places (e.g. malls, stations) indicate that the more public a place is, the less would the subject play there. Only 7 subjects would play the game in public with friends or strangers, and 8 subjects would not play at all. Some of these subjects stated that they did not want to behave in an awkward way or disturb other people in public.

Attracting attention seems to be less desired for the physical interaction with public displays than a loss of privacy, as the last question indicates. On a Likert-scale from 1 ("not at all") to 5 ("very much"), the subjects stated that they were not much concerned about their privacy if they played the Whacka-Mole game in private $(\mathrm{m}=1.1 ; \mathrm{sd}=0.2)$, semi-public $(\mathrm{m}=$ $2.0 ; \mathrm{sd}=0.8)$ or public places $(\mathrm{m}=2.7 ; \mathrm{sd}=1.5)$.

\section{DISCUSSION AND CONCLUSION}

In this paper, we have investigated the touch-like interaction with NFC-tags as a means for direct interaction between mobile devices and public displays. We have adopted the existing technology of dynamic NFC-displays for a game to assess the interaction with NFC-based physical UIs and to evaluate its usability and user acceptance. The Whack-a-Mole prototype showed that the physical interaction between mobile devices and NFC-tags can be used to implement direct interactions with large screens and their content and to distinguish multiple users at the same time.

The evaluation of the prototype showed that the interaction with a grid of NFC-tags works well enough for a gaming application. The tags were recognized with a rate of almost $70 \%$, leaving room for technical improvements. Despite this inaccuracy of the tag recognition, the physical interaction with the dynamic NFC-display was well received. Its playfulness and ease of use seemed to compensate the sub-optimal tag recognition, which can easily cause frustration, especially during a game. The study also indicated that the subjects prefer to use dynamic NFC-displays in private or semi-public places where their interactions attract less attention. The more public a place was, the more willing were the subjects to interact with strangers. However, as we conducted the study under lab conditions, this last result requires verification from further studies in the real world.

Although the evaluation of the Whack-a-Mole prototype advocates the use of dynamic NFC-displays, it is only a prototyping technology for the emulation of direct mobile interactions with public displays and their content. Adopting dynamic NFC-displays for a game that includes a certain amount of physical performance in real time clearly showed the limits of mobile interaction with NFC. The recognition of NFC-tags can still be improved, e.g., with smaller tags or mobile devices that can read multiple tags at the same time. The use of overlapping tags allowed to create a completely interactive surface but also prohibited a better tag recognition rate. Future work on NFC-based physical UIs can explore better technical solutions for their setup, e.g. with smaller, 
adjacent tags that do not overlap, and compare them in more sophisticated experiments. Dynamic NFC-displays can also only be used for the interaction with screens of a certain size and at a close distance. The interaction with really large displays over greater distances, e.g. in stadiums or at public viewings, should rely on other technologies, e.g. based on the recognition of phonecam images [3].

Finally, the presented results have been elicited with a gaming use case whose specific requirements influenced the design of the application and its evaluation. For example, the game was designed for a certain performance-oriented interaction - touching as many NFC-tags as possible within a short time. This design is suitable to push the technical limits of mobile interaction with NFC, but is not applicable to other use cases, e.g. maps or information retrieval systems, which support other ways of interaction. Therefore, only some of the presented results can be applied to other applications that build upon dynamic NFC-displays or other NFC-based physical UIs. In order to provide more general guidelines for the development of such applications, future work has to broaden the range of applications for this technology, investigate new use cases, compare different UI designs and evaluate the interaction with them.

\section{REFERENCES}

[1] Ballagas, R., Borchers, J., Rohs, M., and Sheridan, J. G. 2006. The Smart Phone: A Ubiquitous Input Device. IEEE Pervasive Computing 5, 1 (Jan. 2006), 70 - 77.

[2] Boring, S., Jurmu, M., and Butz, A. 2009. Scroll, tilt or move it: using mobile phones to continuously control pointers on large public displays. In Proc. of OZCHI '09, vol. 411. ACM, New York, NY, 161-168.

[3] Boring, S., Baur, D., Butz, A., Gustafson, S., and Baudisch, P. 2010. Touch projector: mobile interaction through video. In Proc. of CHI '10. ACM, New York, NY, 2287-2296.

[4] Broll, G., Rukzio, E., Paolucci, M., Wagner, M., Schmidt, A., and Hussmann, H. 2009. Perci: Pervasive Service Interaction with the Internet of Things. IEEE Internet Computing 13, 6 (Nov. 2009), 74-81.

[5] Broll, G., Graebsch, R., Holleis, P., and Wagner, M. 2010. Touch to play: mobile gaming with dynamic, NFC-based physical user interfaces. In Proc. of MobileHCI '10. ACM, New York, NY, USA, 459-462.

[6] Coulton, P., Rashid, O., and Bamford, W. 2006. Experiencing 'Touch' in Mobile Mixed Reality Games. In Proc. of the $4^{\text {th }}$ International Conference in Computer Computer Game Design and Technology, Nov 15 - 16, Loverpool, UK.

[7] Garner, P., Rashid, O., Coulton, P., and Edwards, R. 2006. The mobile phone as a digital SprayCan. In Proc. of ACE '06. ACM, New York, NY, USA

[8] Hardy, R. and Rukzio, E. 2008. Touch \& interact: touch-based interaction of mobile phones with displays. In Proc. of MobileHCI '08. ACM, New York, NY, 245-254.

[9] NTT DOCOMO i-mode Felica, www.nttdocomo.co.jp/ english/service/imode/make/content/felica/index.html

[10] Oyster Card website: https://oyster.tfl.gov.uk/oyster/entry.do

[11] Ramírez-González, G., Muñoz-Organero, M., Kloos, C. D., and Astaiza, Á. C. 2008. Exploring NFC interactive panel. In Proc. of Mobiquitous 2008. Dublin, Ireland, July 21 - 25, 2008.

[12] Rashid, O., Bamford, W., Coulton, P., Edwards, R., and Scheible, J. 2006. PAC-LAN: mixed-reality gaming with RFID-enabled mobile phones. Comput. Entertain. 4, 4 (Oct. 2006), 4

[13] Reilly, D., Welsman-Dinelle, M., Bate, C., and Inkpen, K. 2005. Just point and click?: using handhelds to interact with paper maps. In Proc. of MobileHCI '05, vol. 111. ACM, 239-242.

[14] Riekki, J., Salminen, T., and Alakärppä, I. 2006. Requesting Pervasive Services by Touching RFID Tags. IEEE Pervasive Computing 5(1): 4046.
[15] Sánchez, I., Riekki, J., and Pyykknen, M. 2008. Touch \& control: Interacting with services by touching RFID tags. In Proc. of IWRT 08, June 12-13 2008.

[16] Seewoonauth, K., Rukzio, E., Hardy, R., and Holleis, P. 2009. Touch \& connect and touch \& select: interacting with a computer by touching it with a mobile phone. In Proc. of MobileHCI '09. ACM, New York, NY, $1-9$.

[17] SmartTouch website. www.smarttouch.org

[18] Vetter, J., Hamard, J., Paolucci, M., Rukzio, E., and Schmidt, A. 2007. Physical Mobile Interaction with Dynamic Physical Objects. Demo at MobileHCI'07, Singapore, September 9, 2007.

[19] Want, R., Fishkin, K. P., Gujar, A., and Harrison, B. L. 1999. Bridging physical and virtual worlds with electronic tags. In Proc. of CHI '99. ACM, 370-377.

[20] Want, R. 2006. An Introduction to RFID Technology. IEEE Pervasive Computing 5, 1 (Jan. 2006), 25. 